\title{
Scalable Zonotope-Ellipsoid Conversions using the Euclidean Zonotope Norm
}

\author{
Victor Gaßmann and Matthias Althoff
}

\begin{abstract}
Set-based computations become increasingly popular for safety-critical systems to ensure properties of controllers and observers. To efficiently compute various set operations, one often uses different set representations and conversions between them. Two popular set representations, for which scalable conversion algorithms do not yet exist, are zonotopes and ellipsoids. We provide computational approaches for all four conversion cases, i.e., overapproximations and underapproximations from zonotopes to ellipsoids and vice versa. By using upper bounds on the maximum and lower bounds on the minimum Euclidean norm of a given zonotope, our approaches have polynomial complexity and thus can be used for high-dimensional spaces. We show that the tightness of our approaches directly depends on the tightness of the Euclidean norm. Numerical experiments demonstrate the usefulness of our proposed methods.
\end{abstract}

\section{INTRODUCTION}

Since the introduction of zonotopes for reachability analysis of discrete-time linear systems [1], they have become increasingly popular for computing reachable states of further system classes [2]-[5]. While zonotopes are closed under linear transformations and Minkowski sum, the latter increases the representation size. In contrast, ellipsoids are not closed under Minkowski sum; nevertheless, they are used in state estimation [6], [7] and for the computation of invariant sets [8], as they provide a very compact representation of highdimensional spaces.

For certain applications, conversions between state representations might be necessary, e.g., when intersecting an ellipsoid with a zonotope. In [9], an approach for computing both the minimum-volume ellipsoid enclosing a polytope as well as a maximum-volume ellipsoid inscribed in a polytope is presented. A more sophisticated approach for computing the minimum-volume enclosing ellipsoid is given in e.g., [10], while an approximate solution is described in [11]. However, the computation of minimum-volume and maximum-volume enclosing ellipsoids requires the availability of a vertex representation or a half-space representation, making it not scalable for many dimensions [12].

Therefore, we propose novel, more scalable approaches for conversions between ellipsoids and zonotopes. We derive methods to compute overapproximations and underapproximations for both conversion directions. Because exact

This work has been financially supported by the European Commission project justITSELF under grant number 817629 .

The authors are with the Department of Informatics, Technische Universität München, Boltzmannstr. 3, 85748 Garching, Germany (email: $\{$ victor.gassmann, althoff\}@in.tum.de)

Digital Object Identifier: 10.23919/ACC45564.2020.9147938 methods optimizing the volume of sets are either not available or require the computation of the half-space or vertex representation of a zonotope, we investigate approximations based on the maximum and minimum zonotope norm.

This paper is organized as follows: Sec. II introduces some notation as well as necessary definitions and basic operations. We provide new methods for conversions between zonotopes and ellipsoids for all four cases: First, we present the enclosing ellipsoid in Sec. III as well as inscribed zonotopes in Sec. IV as they both are dependent on the maximum zonotope norm. In Sec. V we present a new method for inscribing an ellipsoid into a zonotope and enclosing an ellipsoid by a zonotope, as these tasks are dependent on the minimum zonotope norm. We conclude with results in Sec. VI and a summary in Sec. VII.

\section{Notation AND PRELIMINARIES}

Let us denote with $\mathbb{N}_{+}, \mathbb{R}, \mathbb{R}_{+}$, the set of positive natural, real and positive real numbers. Sets are denoted by upper-case caligraphic letters, e.g., $\mathcal{A}$, matrices by uppercase letters, e.g., $A$, and vectors and scalars are given by lower-case letters, e.g., $a$. Also we require $\mathcal{B}=\{-1,1\}$. Let $\mathcal{W}^{o}, o \in \mathbb{N}_{+}$be the $o$-ary Cartesian product of a set $\mathcal{W}$, i.e., $\mathcal{W}^{o}=\mathcal{W} \times \cdots \times \mathcal{W}$ (o times). A scalar vector component for a $q$-dimensional vector $v$ is denoted by $v_{i}$, $i \in\{1, \ldots, q\}, q \in \mathbb{N}_{+}$. For a given $q$-dimensional vector $w$, we define for $r<q\left(r, q \in \mathbb{N}_{+}\right) w_{1: r}:=\left[\begin{array}{lll}w_{1} & \cdots & w_{r}\end{array}\right]^{T}$. The $i$-th column of a matrix $Q$ is denoted by $q_{i}$. The vector $\mathbb{1}$ denotes the vector of ones and $I$ the identity matrix, $e_{i}$ the $i$-th column of $I$ and $0_{r \times r}$ the $r \times r$ zero matrix. Matrices with no indication of dimension are assumed to be of the appropriate dimension given the current context. The trace of two matrices of appropriate dimension is denoted by $\langle A, B\rangle=\operatorname{trace}\left(A^{T} B\right)$. The positive (semi-)definiteness of a matrix $A$ is denoted by $A \succ(\succeq) 0$. When talking about norms, we will assume the Euclidean norm unless stated otherwise. The absolute value of each component of a vector $x$ is denoted by $|x|$. Lastly, whenever the argument $y$ of a function $f(x, y)$ is irrelevant, we replace it by $(\cdot)$.

Next, we define zonotopes and ellipsoids as well as some basic operations on them.

Definition 1 (Zonotope)

A full-dimensional zonotope is given by

$$
\mathcal{Z}(G, \bar{z}):=\left\{z \in \mathbb{R}^{n}|z=G x+\bar{z},| x \mid \leq 1\right\},
$$

where $G \in \mathbb{R}^{n \times m}$ is the $n$-dimensional generator matrix consisting of $m$ generators with $n \leq m$ and $\operatorname{rank}(G)=n$, and $\bar{z} \in \mathbb{R}^{n}$ is the center. 
A given zonotope $\mathcal{Z}$ can equivalently be represented using its vertex representation $\mathcal{Z}=$ $\left\{z \in \mathbb{R}^{n} \mid z \in\right.$ convhull $\left.\left(v^{(1)}, \ldots, v^{(N)}\right)\right\}$, where $v^{(i)} \in \mathbb{R}^{n}$, $i \in\{1, \ldots, N\}$ denotes one of the $N$ vertices of $\mathcal{Z}$ and convhull $(\cdot)$ is the convex hull operation. Alternatively, $\mathcal{Z}$ can also be described using its half-space representation $\mathcal{Z}=\left\{z \in \mathbb{R}^{n} \mid A z \leq b\right\}, A \in \mathbb{R}^{q \times n}, b \in \mathbb{R}^{q}$.

Definition 2 (Ellipsoid)

A full-dimensional ellipsoid is defined by

$$
\mathcal{E}(E, \bar{e}):=\left\{x \in \mathbb{R}^{n} \mid(x-\bar{e})^{T} E^{-1}(x-\bar{e}) \leq 1\right\},
$$

where $E \in \mathbb{R}^{n}$ is the shape matrix, $E \succ 0$, and $\bar{e} \in \mathbb{R}^{n}$ is the center.

Note that from Def. 2, we can conclude that the shape matrix $E$ always has an inverse. We simply write $\mathcal{Z}$ (or $\mathcal{E}$ ) whenever the arguments are clear from the context or are of no relevance.

\section{Lemma 1 (Linear Transformation of a Zonotope)}

The linear transformation $\phi: x \mapsto T x, T \in \mathbb{R}^{n \times n}$, of $a$ given zonotope $\mathcal{Z}(G, \bar{z})$ is given by

$$
T \mathcal{Z}:=\left\{t \in \mathbb{R}^{n} \mid t=T z, z \in \mathcal{Z}\right\}=\mathcal{Z}(T G, T \bar{z}) .
$$

Proof: Follows directly from Def. 1.

For this paper, it suffices to assume that each linear transformation of an ellipsoid is non-singular, i.e., the matrix associated with the linear transformation is invertible.

Lemma 2 (Linear Transformation of an Ellipsoid)

The non-singular linear transformation, defined by $\phi: x \mapsto$ $T x, T \in \mathbb{R}^{n \times n}$, of a given ellipsoid $\mathcal{E}(E, \bar{e})$ is

$$
T \mathcal{E}:=\left\{t \in \mathbb{R}^{n} \mid t=T x, x \in \mathcal{E}\right\}=\mathcal{E}\left(T E T^{T}, T \bar{e}\right) .
$$

Proof: Plugging $\tilde{x}=T x$ into Def. 2 yields $\left(T^{-1} \tilde{x}-\bar{e}\right)^{T} E^{-1}\left(T^{-1} \tilde{x}-\bar{e}\right) \leq 1$. After factoring out $T^{-1}$, we have $(\tilde{x}-T \bar{e})^{T}\left(T E T^{T}\right)^{-1}(\tilde{x}-T \bar{e}) \leq 1$, which is the desired result.

For later derivations we also define the squared maximum and minimum norm of a zonotope.

Definition 3 (Squared Maximum Zonotope Norm)

The squared maximum norm from any point on the boundary of a zonotope $\mathcal{Z}(G, \bar{z})$ to its center $\bar{z}$ is defined by

$$
\lceil\mathcal{Z}(G, \bar{z})\rceil^{2}:=\max _{|u| \leq 1} u^{T} G^{T} G u \text {. }
$$

\section{Definition 4 (Squared Minimum Zonotope Norm)}

The squared minimum norm from any point on the boundary of a zonotope $\mathcal{Z}(G, \bar{z})$ to its center $\bar{z}$ is defined by

$$
\lfloor\mathcal{Z}(G, \bar{z})\rfloor^{2}:=\min \left(b^{2}\right)
$$

where $\left\{z \in \mathbb{R}^{n} \mid A z \leq b\right\}, A \in \mathbb{R}^{q \times n}, b \in \mathbb{R}^{q}$, is the halfspace representation of $\mathcal{Z}(G, 0), b^{2}$ is to be understood component-wise and $\min \left(b^{2}\right)$ is the minimum of all elements of $b^{2}$.

Geometrically, this minimum norm is equal to the radius of the largest $n$-ball that is still enclosed by $\mathcal{Z}$. This result can be obtained as follows: The radius of the maximum-volume inscribed ball of $\mathcal{Z}(G, 0)$ ( [9, Sec. 8.4.2], also see Sec. V) is obtained by

$$
\begin{aligned}
s^{*}= & \underset{s \in \mathbb{R}_{+}}{\arg \max } s, \\
& \text { s.t. }\left\|s I a_{i}\right\| \leq b_{i}, \forall i \in\{1, \ldots, q\},
\end{aligned}
$$

with $a_{i}^{T}$ being the $i$-th row of $A$ and where by definition of this ball we have $\lfloor\mathcal{Z}\rfloor=s^{*}$. If we assume that $a_{i}$ is normed, i.e., $\left\|a_{i}\right\|_{2}=1$, then

$$
\begin{aligned}
& \left\{s \in \mathbb{R}_{+} \mid\left\|s I a_{i}\right\|_{2} \leq b_{i}, \forall i \in\{1, \ldots, q\}\right\} \\
\Leftrightarrow \quad & \left\{s \in \mathbb{R}_{+} \mid s^{2} \mathbb{1} \leq b^{2}\right\},
\end{aligned}
$$

and an equivalent representation of (7) is

$$
s^{* 2}=\min \left(b^{2}\right) .
$$

Together with $\lfloor\mathcal{Z}\rfloor^{2}=s^{* 2}$, this yields the result from Def. 4 .

\section{ENCLOSING ELLIPSOID}

In this section, we overapproximate a given zonotope with a minimum-volume ellipsoid.

\section{A. Minimum-Volume Enclosing Ellipsoid}

We wish to compute the minimum-volume enclosing ellipsoid $\mathcal{E}\left(E_{\min }, \bar{e}_{\min }\right)$ of a given zonotope $\mathcal{Z}(G, \bar{z})$. Due to the symmetric properties of zonotopes, it is intuitive that $\bar{e}_{\min }=$ $\bar{z}$. Assuming that the $N \in \mathbb{N}_{+}$vertices of the zonotope are denoted by $\left\{v_{i}\right\}_{i=1}^{N}$, the minimum-volume enclosing ellipsoid of $\mathcal{Z}$ (see e.g., [9, Sec. 8.4.1]) is $\mathcal{E}\left(E_{\min }, \bar{z}\right)$, with $E_{\text {min }}$ calculated as

$$
\begin{aligned}
E_{\min }= & \underset{E \succ 0}{\arg \min }-\log \operatorname{det} E, \\
& \text { s.t. }\left(v_{i}-c\right)^{T} E^{-1}\left(v_{i}-c\right) \leq 1, \forall i \in\{1, \ldots, N\} .
\end{aligned}
$$

Note that there are more sophisticated ways of solving (11) (e.g., [13]), but those methods also require a vertex representation, which is infeasible for high-dimensional problems (see [12, Theorem 2.5]).

a) Remark: For the special case $n=m$, we have that $G$ has an inverse since we assumed that $\mathcal{Z}$ is a full-dimensional zonotope. Then, we can transform $\mathcal{Z}$ into a hypercube, i.e.,

$$
\mathcal{Z}(I, \hat{z})=G^{-1} \mathcal{Z}(G, \bar{z})
$$

The minimum-volume enclosing ellipsoid of $\mathcal{Z}(I, \hat{z})$ is $\mathcal{E}(n I, \hat{z})$ since the minimum-volume ellipsoid that covers a $n$-dimensional hypercube is the hyper-sphere with radius $\sqrt{n}[14,4.1]$. Applying the inverse, the minimum-volume enclosing ellipsoid of $\mathcal{Z}(G, \bar{z})$ is $\mathcal{E}\left(n G G^{T}, \bar{z}\right)$. Hence, we subsequently assume that $m>n$. 


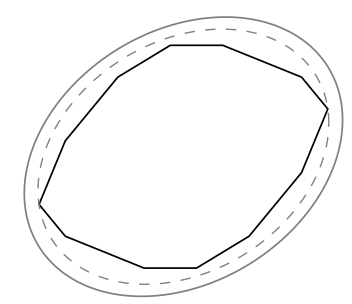

Fig. 1: The given zonotope (black) and the overapproximative ellipsoids using the variation of Goffin's algorithm from [14] (gray) as well as the approach from theorem 1 (dashed gray).

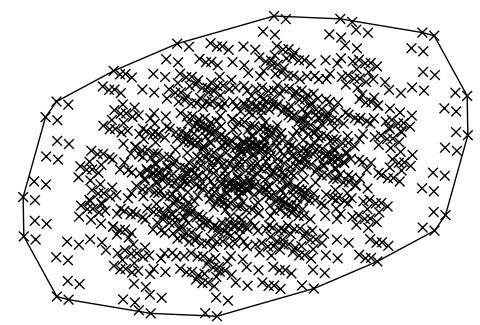

Fig. 2: Distribution of all vertex candidate points (black crosses) for a random zonotope (black border) with $G \in \mathbb{R}^{2 \times 10}$

b) Remark: An approach to compute an approximation to the minimum-volume enclosing ellipsoid (also called Löwner-John ellipsoid) of a zonotope using a variation of Goffin's algorithm is provided in [14]. However, the resulting ellipsoid is not ideal for our purposes as can be seen in Fig. 1 for the zonotope $\mathcal{Z}(G, \bar{z})$ with

$$
G=\left[\begin{array}{ccccc}
-2 & 0 & 1 & -2 & 1 \\
-1 & -2 & 0 & 0 & -1
\end{array}\right], \bar{e}=\left[\begin{array}{l}
-1 \\
-1
\end{array}\right]
$$

which is not very tight. Therefore, we present new approaches.

\section{B. Fitting an Initial Guess}

Fig. 1 shows that the alignment given by the initial guess of $[14$, Sec. 4.1], i.e.,

$$
\mathcal{E}\left(E_{0}, \bar{z}\right)=\mathcal{E}\left(m G G^{T}, \bar{z}\right),
$$

appears to be promising: Let a zonotope $\mathcal{Z}(G, \bar{z})$ and $L=G\left[\begin{array}{ll}B & -B\end{array}\right]$ be given, where $\left[\begin{array}{ll}B & -B\end{array}\right]$ contains all $2^{m}$ possible combinations of $\mathcal{B}^{m}$. For illustration purposes, Fig. 2 depicts the distribution of those points for a random zonotope with $G \in \mathbb{R}^{2 \times 10}$. A Principle Component Analysis (PCA) on the samples (column vectors) of $L$ results in a mean of 0 due to the symmetry of $\left[\begin{array}{ll}B & -B\end{array}\right]$. One way to construct $B \in \mathcal{B}^{m \times 2^{m-1}}$ is to start each row with +1 and alternate the sign for row $i \in\{1, \ldots, m\}$ every $2^{m-1-(i-1)}$ entries. Consequently, $b_{i}^{T} b_{j}=0, \forall i, j \in\{1, \ldots, m\}, i \neq j$, for any two rows $b_{i}, b_{j}$ of $B$. Thus, the corresponding covariance matrix $\bar{L}=\frac{1}{2^{m}-1} L L^{T}=\frac{2}{2^{m}-1} G\left(B B^{T}\right) G^{T}=$ $c G G^{T}$ with $c \in \mathbb{R}_{+}$is (up to a factor) identical to the initial guess provided in (13). Therefore, the initial guess can be interpreted as an ellipsoid which is oriented according to the principal component axes of all samples.
Next, we show how we can use $\lceil\mathcal{Z}(G, \bar{z})\rceil^{2}$ (see Def. 3) to compute the desired shrinking factor. According to Def. 3 , the value of the maximum norm is

$$
\max _{u \in\{-1,1\}^{m}} u^{T} G^{T} G u .
$$

The following proposition rewrites (14) into a mixed integer quadratic program (MIQP).

\section{Proposition 1}

Given $\mathcal{Z}(G, \bar{z}), G \in \mathbb{R}^{n \times m}$, we have

$$
\lceil\mathcal{Z}(G, \bar{z})\rceil^{2}=-\min _{u \in\{-1,1\}^{m}} u^{T} M u+\lambda_{\max } m,
$$

where $M=-\left(G^{T} G-\lambda_{\max } I\right) \succeq 0$ and $\lambda_{\max }$ is the maximum eigenvalue of $G^{T} G$.

Proof: From $u^{T} u=m$ follows that $u^{T} G^{T} G u=$ $u^{T}\left(G^{T} G-\lambda_{\max } I\right) u+\lambda_{\max } m$. Because $G^{T} G \succeq 0$, using $M \succeq 0$ together with Def. 3 provides the desired result $\max _{u \in\{-1,1\}^{m}} u^{T} G^{T} G u=-\min _{u \in\{-1,1\}^{m}}\left\{u^{T} M u\right\}+$ $\lambda_{\max } m$.

Since it is easier to work with hyper-spheres instead of ellipsoids, we transform both $\mathcal{E}$ and $\mathcal{Z}$, using the nonsingular transformation matrix $T=E_{0}^{-\frac{1}{2}}$ to $\mathcal{Z}\left(G_{t}, \hat{z}\right)=T \mathcal{Z}$ and $\mathcal{E}(I, \hat{z})=T \mathcal{E}$ so that $\mathcal{E}(I, \hat{z})$ is the $n$-dimensional unit hyper-sphere with center $\hat{z}$. As a relation between the transformed and original space, we also have

$$
\mathcal{Z} \subseteq \mathcal{E} \Leftrightarrow T \mathcal{Z} \subseteq T \mathcal{E}=\mathcal{E}(I, \hat{z}) .
$$

Hence $\mathcal{Z}\left(G_{t}, \hat{z}\right) \subseteq \mathcal{E}(I, \hat{z})$ if $\left\lceil\mathcal{Z}\left(G_{t}, \hat{z}\right)\right\rceil \leq 1$, since $\mathcal{E}(I, \hat{z})$ has a radius of 1 and $\mathcal{Z}\left(G_{t}, \hat{z}\right)$ can only be contained if the maximum norm is less than 1 .

Proposition 1 is a MIQP and thus in the complexity class NP [15]. However, it has been shown that (14) is of polynomial complexity $\mathcal{O}\left(m^{n-1}\right)$ if the rank of $G_{t}^{T} G_{t}$ is fixed [16]. That said, once $m$ and $n$ become larger, this approach also does not scale well due to its polynomial complexity with the large exponent $n-1$. Therefore, we calculate an upper bound on $\lceil T \mathcal{Z}\rceil^{2}$. Using Def. 3, we can write

$$
p^{*}=\max _{u_{i}^{2} \leq 1, \forall i \in\{1, \ldots, m\}} u^{T} G_{t}^{T} G_{t} u,
$$

where we used the fact that $\left\{u \in \mathbb{R}^{m}|| u \mid \leq 1\right\}=$ $\left\{u \in \mathbb{R}^{m} \mid u_{i}^{2} \leq 1, \forall i \in\{1, \ldots, m\}\right\}$. Its dual problem induces the following lemma.

Lemma 3 (Upper Bound on Maximum Zonotope Norm) Given $\mathcal{Z}(G, \bar{z})$, the dual problem given by

$$
\begin{aligned}
& d^{*}=\min _{\lambda \geq 0} \mathbb{1}^{T} \lambda, \\
& \text { s.t. } \operatorname{diag}(\lambda)-G^{T} G \succeq 0,
\end{aligned}
$$

with $\lambda \in \mathbb{R}^{m}$, provides an upper bound $d^{*} \geq\lceil\mathcal{Z}\rceil^{2}$.

Proof: The result follows immediately from $\min (\cdot)=$ $-\max -(\cdot)$ and the weak-duality-theorem.

The dual (18) is a semi-definite program and thus has a polynomial worst-case complexity [17] so that lemma 3 scales well. Note that other approaches, e.g., [18], are even 
more efficient, but much more complicated to implement. Since lemma 3 is not the bottleneck of our approach, we favor simplicity.

Because of the non-convexity of (17), there is a gap between (17) and (18), which is briefly discussed. First, we rewrite (17) (with the knowledge that $u_{i}^{2}=1, \forall i \in$ $\{1, \ldots, m\}$ as the maximum is attained at vertices of the zonotope) as

$$
\begin{array}{ll} 
& \max _{U}\left\langle G_{t}^{T} G_{t} U\right\rangle, \\
\text { s.t. } & U \succeq 0, \\
& \left\langle\operatorname{diag}\left(e_{i}\right) U\right\rangle=1, \forall i \in\{1, \ldots, m\}, \\
& \operatorname{rank}(U)=1,
\end{array}
$$

where $U=u u^{T}$. Dropping the rank constraint gives us a semi-definite relaxation of (17). The dual of this relaxation is identical to (18). Because (19) is a convex problem and because constraint qualifications hold, strong duality holds and therefore the optimal objective value of the dual is equal to that of the primal. Therefore, the discussed duality gap is due to the dropped rank constraint.

We now formulate the main theorem for enclosing ellipsoids.

Theorem 1 (Enclosing Ellipsoid)

Given $\mathcal{Z}(G, \bar{z})$ and the initial ellipsoid $\mathcal{E}\left(E_{0}, \bar{z}\right)$ from (13), an enclosing ellipsoid is obtained by

$$
\mathcal{E}(E, \bar{z})=\mathcal{E}\left(\hat{r} E_{0}, \bar{z}\right)
$$

where $\hat{r}$ is an upper bound on $\left\lceil E_{0}^{-\frac{1}{2}} \mathcal{Z}(G, \bar{z})\right\rceil^{2}$ using lemma 3.

Proof: Transforming both $\mathcal{Z}(G, \bar{z})$ and $\mathcal{E}\left(E_{0}, \bar{z}\right)$ using $T=E_{0}^{-\frac{1}{2}}$, we obtain $\mathcal{Z}\left(G_{t}, \hat{z}\right)=T \mathcal{Z}(G, \bar{z})$ as well as $\mathcal{E}(I, \hat{z})=T \mathcal{E}\left(E_{0}, \bar{z}\right)$. Lemma 3 returns an upper bound $\hat{r}$ of $\left\lceil\mathcal{Z}\left(G_{t}, \hat{z}\right)\right\rceil^{2}$, which itself bounds the norm of every vertex from its center $\hat{z}$. Therefore, $\mathcal{Z}\left(G_{t}, \hat{z}\right) \subseteq \mathcal{E}(\hat{r} I, \hat{z})$, and applying the inverse transform $T^{-1}=E_{0}^{\frac{1}{2}}$ then yields the desired result.

In [19] (for other approaches see e.g., [20]), a result for tightening this duality gap is provided. While for zonotopes where $n \ll m$, the combined approach from [19, Corollary $2 \&$ Lemma 3] is not applicable, the following example demonstrates the usefulness for cases where $n \approx m$.

\section{Example 1}

Given is a zonotope $\mathcal{Z}(G, \bar{z})$

$$
G=\left[\begin{array}{ccccccc}
1 & -2 & 2 & 0 & 3 & 1 & 0 \\
0 & 0 & -1 & -2 & -2 & -1 & 0 \\
-2 & -1 & 0 & 0 & -2 & 1 & 0 \\
1 & -1 & -1 & 1 & -4 & 0 & 5 \\
-2 & 1 & 0 & 0 & 1 & 0 & -3
\end{array}\right]
$$

and $\bar{z}=0_{5 \times 1}$. Using proposition 1, we find the exact value $\lceil\mathcal{Z}(G, \bar{z})\rceil^{2}=231$. The upper bound on the maximum norm via lemma 3 evaluates to $d^{*}=233.250$. We have $n=5$, $m=7$ and thus $r=2$. From [19, Lemma 3], it follows that $\gamma_{r}=0.976$ and thus the improved upper bound is $\gamma=$ 231.932.
Example 1 shows that the upper bound in (18) can be efficiently checked for a zero gap to the optimal solution and potentially tightened whenever the vertex enumeration of an $r$-dimensional zonotope with $m$ generators is feasible. This is especially interesting whenever the number of vertices is not prohibitively large. However, if $m$ is very close to $n$, it might be better to perform an overapproximative order reduction of the given zonotope to order $1(m=n)$ and then directly compute the minimum-volume enclosing ellipsoid as stated at the end of Sec. III-A. Next, we inscribe a zonotope into a given ellipsoid.

\section{INSCRIBED ZONOTOPE}

Now an ellipsoid $\mathcal{E}(E, \bar{e})$ is given and we want to find a zonotope $\mathcal{Z}(G, \bar{z})$ for a given number of generators $m$ such that $\mathcal{Z} \subseteq \mathcal{E}$. Since we can always transform the ellipsoid into a unit hyper-sphere, we only consider the problem of inscribing a zonotope in a given hyper-sphere for now.

Intuitively, a good under-approximation of the unit hypersphere is a zonotope whose generators are equally spaced on its surface. Computing an exact uniform distribution of $m$ points on the surface of the unit hyper-sphere is impossible for arbitrary $m$ (also see [21]), to the best of our knowledge.

However, one can approximate this exact distribution by randomly picking $m$ uniformly distributed points on the surface of the hyper-sphere [22]. While this approach works well for large $m$, in practice its stochastic nature often leads to a non-uniform distribution for smaller $m$. Therefore, one can use deterministic sampling [21] or center points of a partition on the unit hyper-sphere with roughly equal diameters [23]. For the latter approach, the MATLAB toolbox EQSP: Recursive Zonal Sphere Partitioning Toolbox 1 is available. The following lemma proofs that we can approximate the hyper-sphere arbitrarily closely for arbitrary $m$.

Lemma 4 ( [23, Theorem 5.4.1.], [24, 3., Theorem] combined) Let $S \in \mathbb{R}^{n \times n}$ be the collection of $m$ points given by the partitioning algorithm described in [23]. The zonotope $\mathcal{Z}(S, \bar{z})$ approximates a hyper-sphere with radius $r$ increasingly closer (in the Hausdorff distance) for increasing $\mathrm{m}$.

Proof: Without loss of generality, we assume $\bar{z}=0$. With $S$ given by [23], we use [23, Theorem 5.4.1.] as an upper bound on the spherical discrepancy in [24, 3., Theorem], which gives an upper bound on the Hausdorff distance between $\mathcal{Z}(S, 0)$ and $\mathcal{E}\left(r^{2} I, 0\right)$ of the form $\mathcal{O}\left(m^{-\frac{1}{n}}\right)$, which is decreasing in $m$. As [24, 3., Theorem] gives the approximation of the unit-sphere, we scale the generators by the radius $r$ and conclude the proof.

Given the approximation of a hyper-sphere, it is straightforward to inscribe a zonotope into a given ellipsoid.

Theorem 2 (Inscribed Zonotope)

A zonotope $\mathcal{Z}(G, \bar{z})$ with $m$ generators is inscribed in

\footnotetext{
${ }^{1}$ https://de.mathworks.com/matlabcentral/fileexchange/13356-eqsprecursive-zonal-sphere-partitioning-toolbox
} 
$\mathcal{E}(E, \bar{e})$ by

$$
\mathcal{Z}(G, \bar{z})=\mathcal{Z}\left(\frac{1}{\sqrt{\hat{r}}} T^{-1} S, \bar{e}\right)
$$

where

$$
\begin{aligned}
T & =E^{-\frac{1}{2}}, \\
S & =\left[\begin{array}{lll}
v_{1} & \cdots & v_{m}
\end{array}\right],
\end{aligned}
$$

each $v_{i}, \forall i \in\{1, \ldots, m\}$ is a point on the surface of the hyper-sphere, and $\hat{r}$ is an upper bound on $\lceil\mathcal{Z}(S, \cdot)\rceil^{2}$ using lemma 3.

Proof: By choosing $T=E^{-\frac{1}{2}}$, we have $\mathcal{E}(I, \hat{e})=$ $T \mathcal{E}(E, \bar{e})$. From lemma 4, we know that $\mathcal{Z}(S, 0)$ is a good approximation of the unit hyper-sphere with radius $\sqrt{\hat{r}}$. Since $\lceil\mathcal{Z}(S, 0)\rceil^{2} \leq \hat{r}$, we have $\mathcal{Z}(S, \hat{e}) \subseteq \mathcal{E}(\hat{r} I, \hat{e})$ and hence also $\mathcal{Z}\left(\frac{1}{\sqrt{\hat{r}}} S, \hat{e}\right) \subseteq \mathcal{E}(I, \hat{e})$. Applying the inverse transform $T^{-1}$ gives $\mathcal{Z}\left(\frac{1}{\hat{r}} T^{-1} S, \bar{e}\right)$ which concludes the proof.

a) Remark: The upper bound $\hat{r}$ in Theorem 2 can be any upper bound on $\lceil\mathcal{Z}(S, \cdot)\rceil^{2}$ and thus can also be computed using proposition 1.

b) Remark: Since lemma 4 shows that we can approximate the hyper-sphere arbitrarily closely, the procedure in theorem 2 can be made arbitrarily precise.

In the following two sections, we use the minimum squared zonotope norm in order to derive both inscribed ellipsoids as well as enclosing zonotopes.

\section{INSCRIBED ELLIPSOID AND ENCLOSING ZONOTOPE}

Here, we wish to compute the maximum-volume ellipsoid $\mathcal{E}\left(E_{\max }, \bar{z}\right)$ inscribed within a given zonotope $\mathcal{Z}(G, \bar{z})$. In [9, Sec. 8.4.2], a straightforward way of computing this ellipsoid for a polytope with half-space representation $\mathcal{P}=$ $\left\{x \in \mathbb{R}^{n} \mid A x \leq b\right\}$, is given as $\mathcal{E}(B B, \bar{z})$ with

$$
\begin{aligned}
B= & \underset{B \succ 0}{\arg \min }-\log \operatorname{det} B, \\
& \text { s.t. }\left\|B a_{i}\right\|_{2}+a_{i}^{T} \bar{z} \leq b_{i}, \forall i \in\{1, \ldots, N\},
\end{aligned}
$$

where $a_{i}^{T}$ is the $i$-th row of $A \in \mathbb{R}^{q \times n}$ and $b_{i}$ is the $i$-th component of $b \in \mathbb{R}^{q}$. However, the number of half-spaces $q$ for larger $m$ quickly renders (25) intractable [25].

For an approximation of (25), we use the minimum zonotope norm $\lfloor\mathcal{Z}(G, \bar{z})\rfloor$ (see Def. 4), instead of the maximum zonotope norm $\lceil\mathcal{Z}(G, \bar{z})\rceil$, as presented in the next lemma.

\section{Lemma 5 (Lower Bound on Squared Minimum Norm)}

Given is a zonotope $\mathcal{Z}(G, \bar{z})$. For any $\nu \in \mathbb{R}_{+}$such that the test $\nu e_{i} \subseteq \mathcal{Z}, \forall i \in \mathbb{R}^{n}, \nu \in \mathbb{R}_{+}$from [14, Sec. 4.4] is fulfilled, a lower bound $\hat{l} \in \mathbb{R}_{+}$on $\lfloor\mathcal{Z}\rfloor^{2}$ is given by

$$
\hat{l}=\frac{\nu^{2}}{n} \text {. }
$$

Proof: For any $\nu$ fulfilling the test from [14, Sec. 4.4], the tested scaled unit vectors $\nu e_{i}, i \in\{1, \ldots, n\}$, span a rotated hyper-cube. Since a zonotope is a convex set, this hyper-cube is contained. We can inscribe a ball with radius $\frac{\nu}{\sqrt{n}}$ into that hyper-cube such that $\mathcal{E}\left(\frac{\nu^{2}}{n} I, \bar{z}\right) \subseteq \mathcal{Z}$, which concludes the proof.
Before we continue, a quick note about the complexity of the test used in lemma 5 . In order to verify whether a given $\nu$ passes the test, we check whether the $n$ points $\nu e_{i}$, $i \in\{1, \ldots, n\}$, are contained in $\mathcal{Z}$. Checking the containment of a given point $\nu e_{i}$ is efficiently possible, as this reduces to checking the feasibility of a linear program, specifically the feasibility of $\nu e_{i}=G x+\bar{z}$, where $x \in[-1 ; 1]^{m}$ and $G \in \mathbb{R}^{n \times m}$, which is of polynomial complexity. Thus, an instance of this test has complexity $\mathcal{O}\left(n l_{p}\right)$, where $l_{p}$ is the complexity of checking the feasibility of a linear program. Now that we have a constructive way to compute a lower bound on the minimum norm of the zonotope, we are ready to state the main result.

\section{Theorem 3 (Inscribed Ellipsoid)}

Given is a zonotope $\mathcal{Z}(G, \bar{z})$. Let $\mathcal{E}\left(E_{0}, \bar{z}\right)$ and set $E_{0}=$ $G G^{T}$. We obtain the lower bound $\hat{l}$ on $\left\lfloor E_{0}^{-\frac{1}{2}} \mathcal{Z}(G, \bar{z})\right\rfloor^{2}$ from lemma 5. Then, an inscribed ellipsoid is given by

$$
\mathcal{E}(E, \bar{e})=\mathcal{E}\left(\hat{l} E_{0}, \bar{z}\right) .
$$

Proof: For $T=E_{0}^{-\frac{1}{2}}$, we have $\mathcal{Z}\left(G_{t}, \hat{z}\right)=T \mathcal{Z}(G, \bar{z})$. With lemma 5 , we compute a lower bound $\hat{l}$ on the minimum squared norm for $\mathcal{Z}\left(G_{t}, \hat{z}\right)$. Therefore, we know that the hyper-sphere with radius $\sqrt{\hat{l}}$, i.e., $\mathcal{E}(\hat{l} I, \hat{z})$, is still contained in $\mathcal{Z}\left(G_{t}, \hat{z}\right)$. Applying the inverse transform yields the desired result.

Lastly, we enclose a given ellipsoid with a zonotope consisting of $m$ generators, similar to Sec. IV. Since we already know how to compute a lower bound on the squared minimum norm (see lemma 5), and since we have a way to generate a zonotope approximating a hyper-sphere (see lemma 4), we directly state the result.

\section{Theorem 4 (Enclosing Zonotope)}

Let $\mathcal{E}(E, \bar{e})$ be given. Then an enclosing zonotope $\mathcal{Z}(G, \bar{z})$ of dimension $n$ with a given number of generators $m$ is given by

$$
\mathcal{Z}(G, \bar{z})=\mathcal{Z}\left(\frac{1}{\sqrt{\hat{l}}} T^{-1} S, \bar{e}\right),
$$

where

$$
\begin{aligned}
T & =E^{-\frac{1}{2}}, \\
S & =\left[\begin{array}{lll}
v_{1} & \cdots & v_{m}
\end{array}\right],
\end{aligned}
$$

$v_{i}, \forall i \in\{1, \ldots, m\}$ is a point on the surface of the hypersphere and $\hat{l}$ is a lower bound of $\lfloor\mathcal{Z}(S, \cdot)\rfloor^{2}$.

Proof: The proof is analogous to the proof of theorem 2. With $T=E^{-\frac{1}{2}}$, we have $\mathcal{Z}\left(G_{t}, \hat{e}\right)=T \mathcal{Z}(G, \bar{e})$ and $\mathcal{E}(I, \hat{e})=T \mathcal{E}(E, \bar{e})$. Further we know that $\mathcal{E}(I, \hat{e}) \subseteq$ $\mathcal{Z}\left(\frac{1}{\sqrt{\hat{l}}} S, \hat{e}\right)$ due to the definition of $\hat{l}$. Applying the inverse transform $T^{-1}$ then gives the desired result.

\section{RESUlts}

First, note that we do not include results for theorems 2 and 4 , as lemma 4 shows that both theorems in principle allow arbitrary precision for exact maximum and minimum 
norm values, respectively. Since we want to illustrate the performance of theorems 1 and 3 for optimal shrinking factors, we calculate both minimum and maximum zonotope norm exactly, and omit computations using upper and lower bounds due to space limitations.

Furthermore, we denote with $t_{\mathrm{avg}}, t_{\max }$ the average and maximum execution time in seconds, and define $\Delta V:=$ $\left(\frac{\operatorname{vol}(\mathcal{Z})}{\operatorname{vol}(\mathcal{E})}\right)^{\frac{1}{n}}$ where $\operatorname{vol}(\cdot)$ returns the volume and $n$ is the dimension. Further, $\Delta V_{\min }, \Delta V_{\max }$ and $\Delta V_{\text {avg }}$ denote the minimum, maximum, and average volume ratios, respectively. All generator matrices are constructed using the randn function from MATLAB. The shown results are averaged over $N=100$ runs, where the first ten runs for each instance (fixed $m, n$ ) were omitted for the computation of execution times due to startup delays. Lastly, while both theorems 1 and 3 scale beyond what is shown with the usage of upper and lower bounds, the poor scalability of the optimal approaches prevents any high-dimensional computations. That said, the bottom half of Tables I and II contain the highest dimensional results computable using an Intel Xeon Gold $61363.00 \mathrm{GHz}$ processor and $128 \mathrm{~GB}$ of DDR4 $2666 \mathrm{MHz}$ memory. Results that were not computable due to (standard) MATLAB memory limits are denoted by - . These memory issues are caused by the vertex computation done with the MPT3 $^{2}$ toolbox and might be preventable using a different implementation.

All remaining results are computed on a Lenovo T480s with an Intel Core i $8650 \mathrm{U}$ and 24 gigabytes of memory. The proposed methods are all implemented in MATLAB, using the YALMIP modeling framework [26] in conjunction with the Gurobi ${ }^{3}$ and SDPT3 ${ }^{4}$ solver. The CORA toolbox [27] is used for zonotope operations.

Because we used parallel computing for the bottom-half results in tables I and II and because those results were computed using a different CPU, we omit the executing times for these results.

\section{A. Enclosing Ellipsoids}

For an analysis of theorem 1, we look at the tightness of the presented approach by comparing the normalized volume of the resulting ellipsoid and that of the given zonotope. As we have seen, there is no tractable way to compute the optimal ellipsoid that encloses a given zonotope with minimal volume for high-dimensional zonotopes. This, however, implies that we are restricted to rather low dimensionality as otherwise the minimum-volume enclosing ellipsoid of $\mathcal{Z}$, which is required for a comparison, is not computable.

As we saw in Sec. III, $E_{\min }$ is the shape matrix of the minimum-volume enclosing ellipsoid that contains $\mathcal{Z}$. Table I shows a comparison between the ideal enclosing ellipsoid given by (11) and the approach from theorem 1 .

First, we notice that for fixed $m$ and increasing $n$, the optimal ratio $\Delta V$ in both cases decreases, while for fixed

\footnotetext{
${ }^{2}$ https://www.mpt3.org/

${ }^{3}$ http://www.gurobi.com

${ }^{4}$ http://www.math.nus.edu.sg/ mattohkc/sdpt3.html
}

TABLE I: Depicted are the volume ratios $\Delta V_{\min }, \Delta V_{\text {avg }}$, execution times $t_{\mathrm{avg}}, t_{\max }$ using (11) $\left(E_{\min }\right)$ and theorem $1(E)$.

\begin{tabular}{|c|c|c|c|c|c|c|c|}
\hline \multirow{2}{*}{$\begin{array}{l}m \\
n\end{array}$} & & \multicolumn{2}{|c|}{10} & \multicolumn{2}{|c|}{20} & \multicolumn{2}{|c|}{30} \\
\hline & & $E_{\text {min }}$ & $E$ & $E_{\min }$ & $E$ & $E_{\text {min }}$ & $E$ \\
\hline \multirow{4}{*}{2} & $\Delta V_{\text {avg }}$ & 0.935 & 0.889 & 0.959 & 0.923 & 0.966 & 0.937 \\
\hline & $\Delta V_{\min }$ & 0.850 & 0.809 & 0.920 & 0.858 & 0.904 & 0.879 \\
\hline & $t_{\text {avg }}[\mathrm{s}]$ & 0.194 & 0.161 & 0.202 & 0.174 & 0.194 & 0.265 \\
\hline & $t_{\max }[\mathrm{s}]$ & 0.234 & 0.196 & 0.317 & 0.199 & 0.236 & 0.584 \\
\hline \multirow{4}{*}{3} & $\Delta V_{\text {avg }}$ & 0.875 & 0.830 & 0.922 & 0.877 & 0.937 & 0.897 \\
\hline & $\Delta V_{\min }$ & 0.839 & 0.769 & 0.884 & 0.829 & 0.911 & 0.833 \\
\hline & $t_{\text {avg }}[\mathrm{s}]$ & 0.199 & 0.159 & 0.223 & 0.170 & 0.311 & 0.276 \\
\hline & $t_{\max }[\mathrm{s}]$ & 0.237 & 0.194 & 0.272 & 0.193 & 0.466 & 0.578 \\
\hline \multirow{4}{*}{4} & $\Delta V_{\text {avg }}$ & 0.828 & 0.787 & 0.886 & 0.844 & 0.910 & 0.873 \\
\hline & $\Delta V_{\min }$ & 0.781 & 0.741 & 0.865 & 0.805 & 0.890 & 0.835 \\
\hline & $t_{\text {avg }}[\mathrm{s}]$ & 0.220 & 0.164 & 0.544 & 0.170 & 4.506 & 0.276 \\
\hline & $t_{\max }[\mathrm{s}]$ & 0.325 & 0.230 & 0.603 & 0.191 & 4.903 & 0.645 \\
\hline \multirow{2}{*}{5} & $\Delta V_{\text {avg }}$ & 0.779 & 0.746 & 0.855 & 0.817 & - & 0.850 \\
\hline & $\Delta V_{\min }$ & 0.749 & 0.717 & 0.836 & 0.773 & - & 0.826 \\
\hline
\end{tabular}

$n$ and increasing $m$, it increases. Assume that for a fixed $n$, we are given two zonotopes and their respective optimal ellipsoids. We transform for both cases the zonotope and the ellipsoid such that the latter is a hyper-sphere. It is intuitive that a zonotope with more generators can approximate a hyper-sphere better than one with less (see also lemma 4). Therefore, the volume ratio increases for fixed $n$ and increasing $m$. As for decreasing $n$ and fixed $m$ : Intuitively, higher dimensional spaces (and zonotopes) require more generators to achieve the same volume ratio. Therefore, increasing $n$ for fixed $m$ is similar to decreasing $m$ for fixed $n$ and thus yields a similar outcome.

Lastly, while theorem 1 performs worse than (11), we note that the relative difference $\frac{\operatorname{vol}(E)}{\operatorname{vol}\left(E_{\min }\right)}$ for fixed $n$ and increasing $m$ also decreases for the results shown. This might indicate that $\operatorname{vol}(E)$ approaches $\operatorname{vol}\left(E_{\min }\right)$ for very large $m$. This in turn implies that the initial ellipsoid according to (13) for very large $m$ has the same shape as the optimal ellipsoid from (11).

\section{B. Inscribed Ellipsoids}

Next, we analyze the performance of theorem 3, depicted in table II. Instead of approaching $\Delta V=1$ from below, we now only have values greater than 1 .

As before, the volume of the maximum-volume inscribed ellipsoid decreases for fixed $m$ and increasing $n$. Similarly for fixed $n$ and increasing $m$. For detailed reasoning, see the analogous argument in Sec. VI-A.

It is worth to note that the computation times differ substantially, even though both approaches need to compute the half-space representation of the given zonotope. However, the minimum norm follows directly from (6), while (25) requires solving a convex optimization problem.

Also, analogously to Sec. VI-A, we find that $\frac{\operatorname{vol}(E)}{\operatorname{vol}\left(E_{\max }\right)}$ decreases with increasing $m$ for fixed $n$. Again, this might indicate that the initial inscribed ellipsoid (which has the same shape as the initial ellipsoid from (13)) has the same 
TABLE II: Depicted are the volume ratios $\Delta V_{\max }, \Delta V_{\text {avg }}$, execution times $t_{\text {avg }}, t_{\max }$ using $(25)\left(E_{\max }\right)$ and theorem $3(E)$.

\begin{tabular}{|c|c|c|c|c|c|c|c|}
\hline \multirow{2}{*}{$\frac{m}{n}$} & & \multicolumn{2}{|c|}{10} & \multicolumn{2}{|c|}{20} & \multicolumn{2}{|c|}{30} \\
\hline & & $E_{\max }$ & $\boldsymbol{E}$ & $E_{\max }$ & $\boldsymbol{E}$ & $E_{\max }$ & $E$ \\
\hline \multirow{4}{*}{2} & $\Delta V_{\text {avg }}$ & 1.046 & 1.104 & 1.032 & 1.084 & 1.026 & 1.067 \\
\hline & $\Delta V_{\max }$ & 1.101 & 1.328 & 1.069 & 1.218 & 1.059 & 1.133 \\
\hline & $t_{\text {avg }}[\mathrm{s}]$ & 0.187 & 0.010 & 0.187 & 0.001 & 0.186 & 0.001 \\
\hline & $t_{\max }[\mathrm{s}]$ & 0.223 & 0.002 & 0.234 & 0.002 & 0.217 & 0.002 \\
\hline \multirow{4}{*}{3} & $\Delta V_{\text {avg }}$ & 1.090 & 1.207 & 1.065 & 1.131 & 1.053 & 1.107 \\
\hline & $\Delta V_{\max }$ & 1.147 & 1.427 & 1.118 & 1.263 & 1.099 & 1.181 \\
\hline & $t_{\text {avg }}[\mathrm{s}]$ & 0.199 & 0.002 & 0.214 & 0.003 & 0.256 & 0.006 \\
\hline & $t_{\max }[\mathrm{s}]$ & 0.240 & 0.003 & 0.296 & 0.005 & 0.319 & 0.007 \\
\hline \multirow{4}{*}{4} & $\Delta V_{\text {avg }}$ & 1.143 & 1.281 & 1.095 & 1.185 & 1.080 & 1.154 \\
\hline & $\Delta V \max$ & 1.195 & 1.637 & 1.127 & 1.279 & 1.104 & 1.340 \\
\hline & $t_{\text {avg }}[\mathrm{s}]$ & 0.217 & 0.004 & 0.373 & 0.015 & 0.922 & 0.055 \\
\hline & $t_{\max }[\mathrm{s}]$ & 0.264 & 0.004 & 0.434 & 0.016 & 1.017 & 0.064 \\
\hline \multirow{2}{*}{6} & $\Delta V_{\text {avg }}$ & 1.259 & 1.486 & 1.159 & 1.301 & 1.127 & 1.228 \\
\hline & $\Delta V_{\max }$ & 1.332 & 1.932 & 1.200 & 1.769 & 1.154 & 1.455 \\
\hline
\end{tabular}

shape as the maximum-volume inscribed one from (25) for large $m$.

Finally, we note that the minimum-volume enclosing ellipsoid is more expensive to compute than the maximumvolume inscribed ellipsoid, as can be seen from tables I and II. This is due to the well-known fact that computing the vertex representation of a zonotope is generally more expensive than computing the half-space representation.

\section{CONCLUSIONS}

In this work, we derived approaches to compute both underapproximations and overapproximations for ellipsoids and zonotopes. Due to the convex nature of the chosen upper and lower bounds on the maximum and minimum zonotope norm, we are able to perform all four approximations for many dimensions. The accuracy of the proposed methods heavily depends on the tightness of the respective bounds. We note that our results will also be implemented in the next CORA release.

Future work includes tightening of both upper and lower bounds for the maximum and minimum zonotope norm. Specifically, it might be possible to obtain an expression for the minimum zonotope norm that does not depend on the facet description, which in turn might enable a lower bound computation using an approach similar to the duality argument for the upper bound on the maximum zonotope norm.

\section{ACKNOWLEDGMENT}

We gratefully acknowledge financial support by the project justITSELF funded by the European Research Council (ERC) under grant agreement No 817629.

\section{REFERENCES}

[1] W. Kühn, "Rigorously computed orbits of dynamical systems without the wrapping effect," Computing, vol. 61, no. 1, pp. 47-67, 1998.

[2] M. Althoff, O. Stursberg, and M. Buss, "Reachability analysis of nonlinear systems with uncertain parameters using conservative linearization," in 47th IEEE Conference on Decision and Control, 2008, pp. 4042-4048.
[3] M. Althoff, "Reachability analysis and its application to the safety assessment of autonomous cars," Ph.D. dissertation, Technische Universität München, 2010.

[4] E. Asarin, T. Dang, G. Frehse, A. Girard, C. Le Guernic, and O. Maler, "Recent progress in continuous and hybrid reachability analysis," in Joint IEEE Conference on Control Applications, Computer-Aided Control Systems Design Symposium, International Symposium on Intelligent Control, 2006, pp. 1582-1587.

[5] A. Girard, "Reachability of uncertain linear systems using zonotopes," in International Workshop on Hybrid Systems: Computation and Control. Springer, 2005, pp. 291-305.

[6] F. Schweppe, "Recursive state estimation: unknown but bounded errors and system inputs," IEEE Transactions on Automatic Control, vol. 13, no. 1, pp. 22-28, 1968.

[7] D. Bertsekas and I. Rhodes, "Recursive state estimation for a setmembership description of uncertainty," IEEE Transactions on Automatic Control, vol. 16, no. 2, pp. 117-128, 1971.

[8] F. Blanchini, "Set invariance in control," Automatica, vol. 35, no. 11, pp. 1747-1767, 1999.

[9] S. Boyd and L. Vandenberghe, Convex optimization. Cambridge university press, 2004

[10] N. Moshtagh, "Minimum volume enclosing ellipsoid," Convex Optim., vol. 111 , p. $112,2005$.

[11] M. J. Todd and E. A. Yıldırım, "On Khachiyan's algorithm for the computation of minimum-volume enclosing ellipsoids," Discrete Applied Mathematics, vol. 155, no. 13, pp. 1731-1744, 2007.

[12] K. Fukuda, "From the zonotope construction to the Minkowski addition of convex polytopes," Journal of Symbolic Computation, vol. 38, no. 4, pp. 1261-1272, 2004

[13] P. Sun and R. M. Freund, "Computation of minimum-volume covering ellipsoids," Operations Research, vol. 52, no. 5, pp. 690-706, 2004.

[14] M. Černỳ, “Goffin's algorithm for zonotopes," Kybernetika, vol. 48, no. 5, pp. 890-906, 2012.

[15] C. H. Papadimitriou, "On the complexity of integer programming," Journal of the ACM (JACM), vol. 28, no. 4, pp. 765-768, 1981.

[16] J.-A. Ferrez, K. Fukuda, and T. M. Liebling, "Solving the fixed rank convex quadratic maximization in binary variables by a parallel zonotope construction algorithm," European Journal of Operational Research, vol. 166, no. 1, pp. 35-50, 2005.

[17] L. Vandenberghe and S. Boyd, "Semidefinite programming," SIAM review, vol. 38, no. 1, pp. 49-95, 1996.

[18] P. Wang, C. Shen, A. van den Hengel, and P. H. Torr, "Large-scale binary quadratic optimization using semidefinite relaxation and applications," Transactions on Pattern Analysis and Machine Intelligence, vol. 39, no. 3, pp. 470-485, 2016.

[19] U. Malik, I. M. Jaimoukha, G. D. Halikias, and S. Gungah, "On the gap between the quadratic integer programming problem and its semidefinite relaxation," Mathematical programming, vol. 107, no. 3, pp. 505-515, 2006.

[20] G. D. Halikias, I. M. Jaimoukha, U. Malik, and S. Gungah, "New bounds on the unconstrained quadratic integer programming problem," Journal of Global Optimization, vol. 39, no. 4, pp. 543-554, 2007.

[21] A. Yershova and S. M. LaValle, "Deterministic sampling methods for spheres and SO (3)," in Proceedings of the IEEE International Conference on Robotics and Automation, vol. 4. IEEE, 2004, pp 3974-3980.

[22] M. E. Muller, "A note on a method for generating points uniformly on n-dimensional spheres," Communications of the ACM, vol. 2, no. 4 pp. 19-20, 1959.

[23] P. Leopardi, "Distributing points on the sphere," School of Mathematics and Statistics. PhD thesis. University of New South Wales, 2007.

[24] J. Linhart, "Approximation of a ball by zonotopes using uniform distribution on the sphere," Archiv der Mathematik, vol. 53, no. 1, pp. 82-86, 1989.

[25] M. Althoff, O. Stursberg, and M. Buss, "Computing reachable sets of hybrid systems using a combination of zonotopes and polytopes," Nonlinear analysis: hybrid systems, vol. 4, no. 2, pp. 233-249, 2010.

[26] J. Löfberg, "Yalmip : A toolbox for modeling and optimization in MATLAB," in In Proceedings of the CACSD Conference, Taipei, Taiwan, 2004.

[27] M. Althoff, "An introduction to CORA 2015," in Proc. of the Workshop on Applied Verification for Continuous and Hybrid Systems, 2015. 